\title{
The expression and the nuclear activity of the caretaker gene ku86 are modulated by somatostatin
}

\author{
S. Pucci, ${ }^{1}$ E. Bonanno, ${ }^{1}$ F. Pichiorri, ${ }^{1}$ P. Mazzarelli, ${ }^{2}$ L.G. Spagnoli ${ }^{1}$ \\ ${ }^{1}$ Dept. of Biopathology, University of Rome "Tor Vergata", Rome; 'Dept. of Dermatology, \\ University of Naples "Federico II", Naples, Italy
}

(C)2004, European Journal of Histochemistry

Somatostatin is a peptide hormone that exerts antisecretory and antiproliferative activities on some human tumors. The Ku70/86 heterodimer acts as regulatory subunit of the DNA dependent protein kinase and its DNA binding activity mediates DNA double strands breaks repair that is crucial to maintain the genetic integrity of the genome. The activation of the heterodimer regulates cell cycle progression and the activity of nuclear transcription factors involved in DNA replication and cell proliferation. Moreover Ku86 behaves as a receptor for the growth inhibitory tetradecapeptide, somatostatin. Herein we report that somatostatin treatment to a colon carcinoma cell line (Caco-2) inhibits cell growth and, at same time, strongly modulates the activation of Ku70/86 heterodimer and the levels of Ku86 in the nucleus by increasing its specific mRNA level. Our findings are consistent with the hypothesis that somatostatin controls cell cycle progression and DNA repair through a new signalling pathway that involves the regulation of Ku86 level and modulates the Ku70/86 activity in the nucleus.

Correspondence: Prof. L.G. Spagnoli, MD, Dept. of Biopathology, University of Rome "Tor Vergata", via Montpellier 1, 00133 Rome, Italy.

Phone: international +39.06.2023751 or 20903913 .

Fax international +39-06-20902209.

E-mail: spagnoli@uniroma2.it

Key words: Ku70/86, cell cycle, DNA repair, somatostatin, human tumors

Paper accepted on September 22, 2003

European Journal of Histochemistry

2004; vol. 48 issue 2 [Apr-Jun]: 103-110
S omatostatin (SST) is a peptide hormone, which plays an important regulatory role in the endocrine, paracrine and autocrine secretions of several anterior pituitary, and gastrointestinal hormones.

Besides, it has been shown that SST exerts an antiproliferative activity on some human cancer including breast cancer, pancreatic cancer, small cell lung carcinoma and neuroblastoma (De Vita et al., 1997). The biological activity of this peptide is dependent on its interaction with specific receptors, whose structures have recently been elucidated by means of cloning experiments (Yamada et al., 1992). More recently, a Ku subunit, namely Ku86, in addition to its well-known regulatory functions in DNA repair, revealed to behave as a somatostatin receptor in gastric carcinoma cell (Le Romancer, 1994).

It is worth noting that $\mathrm{Ku}$, heterodimer composed by two subunits respectively of 70 and $86 \mathrm{kDa}$, plays fundamental regulatory functions in DNA repair activities, cell growth and survival after damage (Nussenzweig et al., 1996, Jackson, 1995).In particular the Ku70/86 heterodimer is the regulatory subunit of the DNA-dependent protein kinase (DNAPK) and its DNA-binding activity mediates DNA double-strand breaks repair (Jackson, 1995). This protein kinase also plays a critical role in resealing DNA double strand breaks by non homologous end joining in DNA replication and in $V(D) J$ recombination (Nussenzweig et al., 1996). As a subunit of DNA-PK, $\mathrm{Ku}$ is also largely responsible for the DNA-dependent activation of this enzyme. Therefore, the potential role of the kinase is the recruitment and activation by phosporilation of the repair components, as well as signaling of the DNA damage leading to cell cycle arrest and/or apoptosis (Gottlieb et al., 1993, Goudelock et al, 2003).

Recent evidence support the notion that Ku70/86 is involved in the regulation of cell cycle progression, DNA synthesis and repair, and apoptosis induction, 
this latter by the interplay with p53 (Difilippantonio et al., 2000). As a matter of fact studies with $\mathrm{Ku}^{-\mathrm{O}^{--}}$and $\mathrm{Ku}^{-1-}$ mice have suggested a link between $\mathrm{Ku}$ and the control of cell growth. In particular, it has been demonstrated that the inactivation of Ku leads to defective cell cycle progression and a predisposition toward neoplastic transformation and death (Blunt et al., 1995).

Evidences of a link among Ku70/86 heterodimer function, DNA repair and tumor development (Difilippantio et al., 2000, Wilson et al, 2000) further support the notion that Ku86 and 70 behave as caretaker genes belonging to the class of ATM, BRCA1 and BRCA-2 tumor suppressor genes (Kinzler et al., 1997, Li et al. 1998).

In highly aggressive and metastatic breast and bladder human tumors the Ku70/86 heterodimer DNA-repair activity is down regulated, although the level of the proteins is not affected (Pucci et al., 2001). This observation suggests that the inhibition of DNA-repair capability might be due to post-transcriptional mechanisms that could regulate the Ku70/86 DNA repair activity in tumor and in cell cycle progression (Yang, 1999).

Changes in the subcellular localization of Ku can be controlled by various external growth-regulating stimuli that influence the translocation of both Ku70 and Ku86 to the nucleus without forming an heterodimer. These findings are consistent with the participation of $\mathrm{Ku}$ protein in signal transduction system responsive to the inhibitory effect of cell-cell contact on one hand and to cytokines and growthsupportive components of the culture medium on the other (Le Romancer et al., 1994, Fewell et al., 1996).

In order to elucidate if the antiproliferative hormone somatostatin is involved in the regulation of the activity of caretaker genes that have a role in maintaining the genomic stability and in the control of cell cycle progression, we investigated the Ku70/86 DNA binding activity in a colon carcinoma cell line (Caco) treated with the somatostatin peptide.

Therefore, we studied, at different times of culture, the effect of somatostatin treatment on Caco cell proliferation in relation to Ku70/86 heterodimer formation and activation and the influence of this growth regulator factor on p70 and p86 levels in different cell compartments.

\section{Materials and Methods}

\section{Cell culture}

Colon carcinoma cell lines Caco-2 (American Type Culture Collection, ATCC) were seeded at sub confluent density $\left(6 \times 10^{3}\right.$ cells $\left./ \mathrm{cm}^{2}\right)$. A day after seeding cells were treated with somatostatin to a final concentration of $10^{-5} \mathrm{M}$, if incubation time was longer than 12 hrs fresh somatostatin was added every 12 hrs. Untreated cells were used as control. Treated and untreated cells were seeded in triplicate.

Cells, cultured in triplicate, seeded at the density of $6 \times 10^{3}$ cells $/ \mathrm{cm}^{2}$ as above, were counted at day 1 , 2, 3 and 6. Trypan blue staining was performed to evaluate cell death percentage.

\section{Flow cytometry}

Cell cultures, seeded as above described, were incubated with $10^{-5} \mathrm{M}$ somatostain for 4 and 6 hrs; untreated cultures plated at the same conditions were used as control. At the end of the incubation period culture medium was removed, washed with PBS and harvested by trypsinization, fixed in ethylic alcohol at $70^{\circ}$ (30 minutes at room temperature), then washed in PBS and DNA was stained with propidium iodide $(5 \mu \mathrm{g} / \mathrm{mL})$. RNAse A (60 $\mu \mathrm{g} / \mathrm{mL}$ ) was added, and samples were incubated for 15 min. at room temperature in the dark.

All cell suspensions were analysed with a flow cytometer Coulter Epics XL (Coulter Corporation, Hialeah, Florida, USA) equipped with an air-cooled, $15 \mathrm{~mW}$, argon ion laser operating at $488 \mathrm{~nm}$. The forward scatter, the side scatter and the fluorescent intensity and compensation were set using positive controls. The data were analysed using the Listmode Analysis of the Epics CL software and Multycycle software.

\section{Immunocytochemistry}

Cells were seeded on Nunc Lab-Tek ${ }^{\mathrm{TM}}$ II - CC2 ${ }^{\mathrm{TM}}$ Chamber Slide ${ }^{\mathrm{TM}}$ System (NUNC A/S DK-4000 Roskilde, Denmark) at $6 \times 10^{3}$ cells $/ \mathrm{cm}^{2}$ and treated as previously described. Moreover an immunocytochemical observation was performed also at $28 \mathrm{hrs}$ of treatment: i.e. 24 hrs plus 4 hrs.

For immunostaining cells were fixed in formaldeide $4 \%$ in PBS for $10 \mathrm{~min}$. at $4^{\circ} \mathrm{C}$, permeabilized in $0.01 \%$ triton $\mathrm{X}-100,1 \%$ BSA (Sigma., St.Louis, CA, USA) in PBS for $5^{\prime}$ at $40^{\circ} \mathrm{C}$ and incubated with primary antibody antibodies (goat polyclonal IgG anti-Ku70 or Ku86 (Santa Cruz Biotechnologies, 
Inc.), using kit sc1485, sc-14, sc1487 K) for lh at room temperature. Samples were washed twice extensively with $0.5 \%$ Tween-20 in PBS. Samples were incubated with a secondary biotinylated antibody (anti-goat-HRP IgG, Santa Cruz Biotechnologies, Inc) for $30^{\prime}$ and washed for 3 times with PBS $0.5 \%$ Tween and then incubated $30^{\prime}$ with peroxidase labeled streptavidin.

\section{Preparation of cell and nuclear extracts}

Untreated cells and cell treated for 1, 4, 24 hrs were processed for protein extraction. Post-nuclear and nuclear extractions were performed according to Dignam (Dignam, JD, 1983). Cells were washed twice with PBS and centrifuged $5^{\prime}$ at $1100 \mathrm{rpm}$. The pellet was suspended in $0.2 \mathrm{~mL}$ of ice-cold buffer $A$ (HEPES-KOH 10mM, pH7.9, $\mathrm{MgCl}_{2} 1.5 \mathrm{mM}, \mathrm{KCl}$ $10 \mathrm{mM}$, EDTA $1 \mathrm{mM}$ ); cells were allowed to swell on ice for 10 minutes. The homogenate was centrifuged for $1^{\prime}$ min in a microfuge at 10,000 rpm; the post-nuclear extract containing cytoplasmic proteins was carefully removed from the nuclear pellet and stored at $-80^{\circ} \mathrm{C}$.

The nuclear pellet was resuspended in ice-cold $\mathrm{NaCl}$-extraction buffer and incubated on ice for $15^{\prime}$ minutes. Cellular debris was removed by centrifugation for $5^{\prime}$ in a microfuge at 10,000 rpm. The supernatant fraction, containing DNA binding proteins, was stored at $-80^{\circ} \mathrm{C}$. All steps of protein extraction were checked by optical microscope.

Protein content in the cytoplasmic and nuclear extracts was determined in triplicate by Bradford assay (Bio-Rad Protein Assay, Bio-Rad Laboratories, Munchen). Bovine serum albumin was used as standard.

\section{DNA End Binding (Mobility-Shift Gel Electrophor- esis Assay)}

The electrophoretic mobility shift assay (EMSA) was performed as described previously (Pucci et al., 2000). A ${ }^{32}$ P-end labeled 56bp DNA probe as previously described (Pucci et al., 2000), was incubated with nuclear or cytoplasmic extracts for 30' at room temperature in binding buffer, briefly, the DNA binding reactions contained the labeled probe $(50.000$ $\mathrm{cpm})$ and nuclear (2 mg) or cytoplasmic (5 mg) extracts in the presence of the unspecific competitor.

To normalize all nuclear samples, EMSA was performed incubating the nuclear extracts $(3 \mathrm{mg}$ ) with $50.000 \mathrm{cpm} / \mathrm{sample}$ of ${ }^{32} \mathrm{P}$-end labeled $\mathrm{Sp}-1$
Oligonucleotide (Promega Corporation, Madison WI 53711-5399 USA) in binding buffer with 1mg of poly[dI-dC] as unspecific competitor. For gel super shift experiments goat polyclonal anti-Ku70 and anti-Ku86 antibodies (Santa-Cruz Biotechnologies Inc.) were used. Complexes were separated on $6 \%$ no denaturing polyacrylamide gels, in TBE (45 mM Tris-borate, 1 mMEDTA, pH 8.0). Gels were dried and exposed to X-rays films (Amersham-LIFESCIENCE), overnight at $-80^{\circ} \mathrm{C}$. The optical densities were obtained by scanning densitometry. Proof of the specificity of the Ku70/86 binding was obtained by addition of goat polyclonal anti-Ku70 and anti-Ku86 antibodies. In the presence of these antibodies the DNA-end binding were supershifted. The DNA binding activity of all nuclear extracts was normalized for Sp-1 DNA binding activity. All cytoplasmic extracts were tested for the presence of a nuclear specific factor Sp1 end binding to evaluate the possibility of a nuclear contamination in the cytoplasmic extracts.

\section{Western blotting}

Nuclear or cytoplasmic protein extracts (10mg) were denatured in SDS-PAGE loading buffer and separated by $10 \%$ SDS-PAGE. Proteins were transferred to a PVDF membrane (Hybond-P, Amersham-LIFESCIENCE) using an electroblotting apparatus and incubated for $\mathrm{lh}$ at RT with 0, $5 \%$ BSA, (Difco Lab. Detroit, MI, USA). Membranes were stained with Poinceau $S$ dye, to check for equal loading and homogeneous transfer.

Primary antibodies (goat polyclonal IgG antiKu70 or Ku86, Santa Cruz Biotechnologies, Inc, clusterin monoclonal antibody clone 41D, Upstate Biotecnology, Lake Placid, NY) were incubated for Ih at room temperature; samples were washed extensively with $0.5 \%$ Tween-20 and diluted 1:5000 secondary antibody (anti-goat-HRP IgG, Santa Cruz Biotechnologies, Inc) was added in $0.5 \%$ BSA for $1 \mathrm{~h}$ at room temperature. Filters were reprobed with anti $\beta$-actin (Sigma-Aldrich, Saint Louis, Missouri 63103 USA) or anti-Sp-1 (Santa Cruz Biotechnologies, Inc.) mouse IgG1 monoclonal antibodies, to normalize respectively cytoplasmic or nuclear protein levels.

Filters were washed and developed using an enhanced chemiluminescence system (ECL, Amersham-Pharmacia Biotech). The optical densities were obtained by scanning densitometry, after normalization for nuclear or cytoplasmatic housekeep- 
ing gene product ( $\mathrm{Sp}-1$ and $\beta$-actin) all the experiments were repeated three times and gave similar results.

\section{Immunoprecipitation and native page}

The evaluation of the amount of the heterodimer formation was performed on immunoprecipitate nuclear extracts. After protein determination by Breadford assay (Bio-Rad Protein Assay), immunoprecipitation was conducted by incubating nuclear extracts $(100 \mu \mathrm{g}$ in $250 \mu \mathrm{L})$ in reaction buffer (PBS $+0.05 \%$ NP-40) with $2 \mu \mathrm{g}$ of anti-Ku70 antibody (clone C-19, Santa Cruz Biotechnology). Binding reactions were performed overnight at $4^{\circ} \mathrm{C}$ with constant rocking. Protein $G$ Plus-Agarose (Santa Cruz Biotechnology) then was added (25 $\mu \mathrm{L}$ of resine slurry) followed by incubation for 2 hours at $4^{\circ} \mathrm{C}$ in agitation. Precipitated proteins were collected by centrifugation (13,000 revolutions per minute, $5 \mathrm{~min}$ ) and washed two times with reaction buffer and once with washing buffer (PBS, 0.05\% $\mathrm{NP}-40,0.5 \mathrm{M} \mathrm{NaCl}$ ) to eliminate unspecific bindings. Bound proteins were eluted by boiling for $5 \mathrm{~min}$ in SDS/PAGE loading buffer (Yang C.R. et al., 1999) and analysed by Western blotting.

Native page gel was performed to resolve native nuclear proteins using a discontinuous $7 \%$ polyacrilamide gel according to Davis (Ornstein L. and Davis B.J., 1964). The non-denaturing gel was transferred on PVDF (Hybond-P, Amersham-LIFESCIENCE) membrane and the western blot analysis was performed as described above.

\section{RNA extraction and RT-PCR}

Total RNA samples were prepared from treated and untreated cells using guanidine isothiocianate lyses. A $1 \mu \mathrm{g}$ aliquot of each RNA sample was reverse transcribed and $R T-P C R$ reactions were performed as follows. The cDNA was amplified in a DNA thermocycler (2400; Perkin-Elmer, Norwalk, CT). Briefly, reaction mixtures consisted of $2 \mu \mathrm{L}$ of reverse transcribed RNA in $20 \mu \mathrm{L}$ of RT buffer containing $1.2 \mathrm{mM}$ of each primer, $0.3 \mathrm{ml}$ of $25 \mathrm{mM}$ dNTP and $1.2 \mathrm{U}$ of amplitaq polymerase (PerkinElmer, Norwalk, CT, USA). Twenty-five cycles were performed under the following conditions: $1 \mathrm{~min}$. At $94^{\circ} \mathrm{C}, 30 \mathrm{sec}$ at $60^{\circ} \mathrm{C}$ and $1 \mathrm{~min}$ at $72^{\circ} \mathrm{C}$. The number of cycles was determined according to the necessity to avoid reaction saturability. Reactions were performed in triplicate. The relative amount of the specific amplified for Ku86 has been referred to the amount of the $\beta$ actin. The primers specific for $\beta$ actin, used for PCR analyses, have been previously described (Killen A., 2001). The primers sequences for Ku86 cDNA amplification were: forward $5^{\prime}$ aag aag gcc ttt gag ga, 3'; rewerse 5' gct tcc tca gct gtg aca ga, $3^{\prime}$. The optical densities were obtained by scanning densitometry and analyzed with quantiscan program.

\section{Results}

Effect of somatostatin treatment on cell proliferation

The effect of somatostatin treatment on cell proliferation was observed in a colon carcinoma cell lines (Caco-2) in three independent experiments (Figure 1). Cells seeded at the density of $6 \times 10^{3}$ cells $/ \mathrm{cm}^{2}$ in triplicate in the presence or absence of somatostatin as described in material and methods, were counted after 2, 3, 6 days of culture. As shown in Figure 1 , somatostatin treatment resulted in growth inhibition of the cells treated compared to the control. The proliferation was significantly decreased at day 3 of the treatment in the presence of $10^{-5} \mathrm{M}$ somatostatin (SST). At the day 2 the fresh media was added to the cells cultures in order to examine the effect of media growth factor stimuli on cell proliferation. The proliferation rate of the cell cultures whose media was changed at the day 2 was also determined. The comparison of growth curves of somatostatin treated cells, with or without changing the media, revealed consistently slower proliferation rates (a decrease of $58 \%$ at the day 3 ) and generally lowers growth plateaus than those of untreated cells. In SST treated cells, the inhibition of the proliferation was particularly evident if the medium was changed at day 2 whereas in the controls the proliferation rate was positively influenced by the fresh media. These results demonstrate that the cytostatic effect is related to the somatostatin treatment and does not depend on the decrease of growth - supportive components of the culture medium (serum starvation).

Finally the flow cytometry analysis demonstrated a cell accumulation in G0-GI phase, observed after 4 and 6 hrs of somatostatin treatment, due to the blockade of cell cycle progression (Figure 1, panel $B, C, D, E)$. 

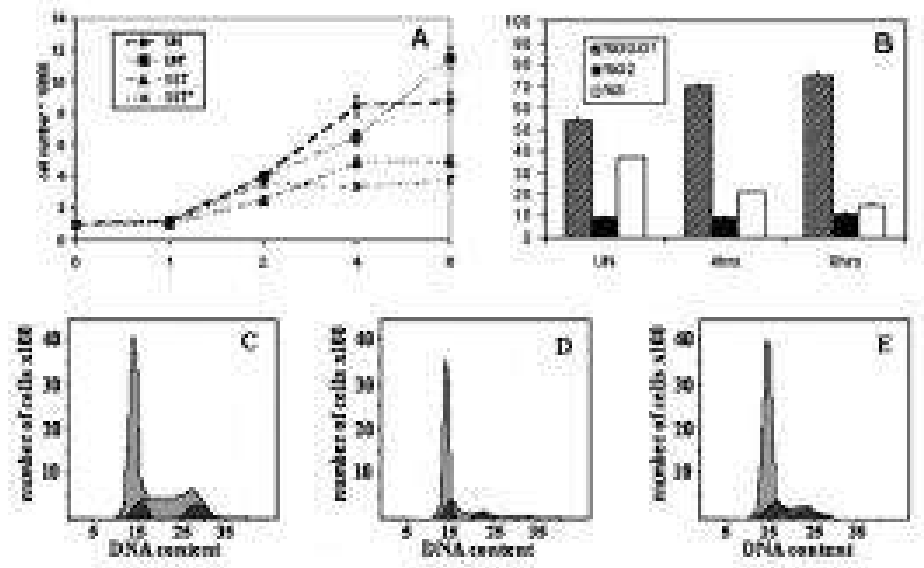

Figure 1. Panel A: growth curves of somatostatin treated Caco cell lines. Experimental groups were untreated cells (UN) and $10 \mathrm{mM}$ SST treated cells both with (*) and without the addition of fresh media at day 2 . Initial cell seeding was $6 \times 10^{3} \mathrm{cell} / \mathrm{cm}^{2}$, cell number represent the total number of cells for well. Panel B, C, D, E represent the flow cytometric analysis: the graph in panel $B$ represents the cell percentage distribution in the cell cycle phases in untreated cells (panel C shows the DNA content), in 4 hrs SST treated cells (panel D for DNA content) and in 6 hrs SST treated cells (panel E for DNA content); bar errors represents C.V. values.

\section{Ku70/86 DNA binding activity is modulated by somatostatin}

To evaluate whether somatostatin treatment could affect directly or indirectly the nuclear Ku70/86 DNA binding activity involved in cell-cycle progression and in DNA replication, nuclear extracts were prepared from untreated and treated Caco cell line at different times of SST treatment.

Figure 2 shows the modulation of the Ku70/86 DNA binding activity in nuclear compartment induced by somatostatin treatment. After $1 \mathrm{hr}$ of SST treatment no differences in DNA binding activity was observed as compared to the control. After 4 hrs the DNA binding activity was increased 10 times as compared to the untreated control. Conversely after 24 hrs there was a decrease of the heterodimer activity ( 2.5 times) induced by somatostatin addition as compared to the DNA binding obtained after 4 hrs of treatment. Proof of the specificity of the Ku70/86 heterodimeric binding was obtained by addition of no cross-reactive goat polyclonal anti-Ku70 or anti-Ku86 antibodies on the same nuclear extracts from Caco cells stimulated for 4hrs with SST (Figure 2, panel B). In the presence of these antibodies the DNA-end binding were supershifted (Figure 2, panel B). This data prove that the interaction of the two subunits is responsible for increase of the DNA binding activity. Moreover, these results demonstrate that somatostatin strongly modulates the Ku DNA binding activity in the nucleus.

\section{Somatostatin affects Ku70/86 heterodimer formation}

To determine whether the Ku DNA binding activation after somatostatin treatment was due to an increased formation of the heterodimer, an immunoprecipitation assay was performed. Nuclear extracts at different time of treatment were prepared as described in materials and methods section.

The evaluation of possible changes in the level of heterodimer formation was performed on immunoprecipitated nuclear extracts by Western blot analysis. After protein determination, immunoprecipitation was conducted by incubating the nuclear protein extracts with anti-Ku70 antibody. Immunoprecipitated aliquots were loaded on SDS denaturing poliacrylamide gel and the co-immunoprecipitated form of Ku86 were determined by western blot analysis. Figure 3 shows the modulating effect of somatostatin treatment on the heterodimer complex formation. An increased amount of interaction between Ku70 and Ku86 subunits was present in the nuclear extracts from cells treated for 4 hrs with SST. This result correlate to the Ku70/86 increased DNA binding activity found in the nucleus at the same time of treatment obtained by EMSA. A further investigation was performed on the same extracts loaded in a native gels as described in materials and methods. Single subunits and heterodimer formation were visualized by Western blot assay. Anti-Ku70 and the no cross reactive antiKu86 were used to determine the presence of the single subunits or the heterodimer complex in the nuclear extracts after four hrs of SST treatment. The policlonal antibody Anti-Ku70 revealed two different bands corresponding to the monomer and heterodimer complex (Figure 3, panel B). At 4 hrs of treatment is clearly evident an increase of the fraction corresponding to the heterodimer complex formation. These results were confirmed, after protein stripping, using anti-Ku86 policlonal antibody 


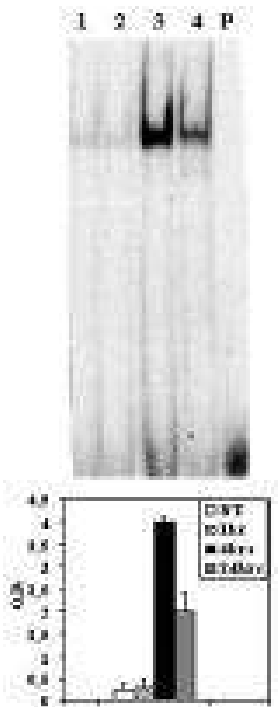

A

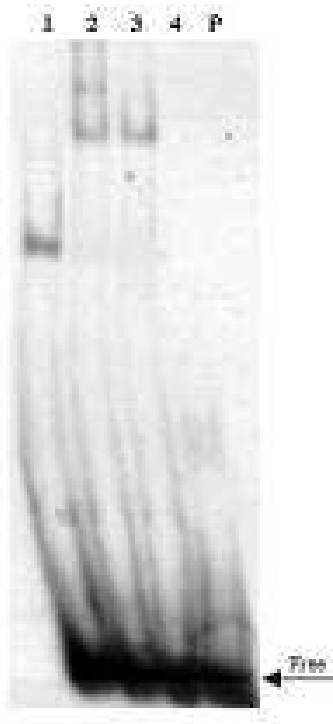

B
Figure 2. Nuclear DNA binding activity of the heterodimer Ku70/86 induced by somatostatin treatment. The kinetic of appearance of ku 70/86 inducibile DNA binding activity was analyzed on a non denaturing $6 \%$ polyacrylamide gel in nuclear extracts (panel A) from untreated cells (lane 1) and cells treated with somatostatin respectively for $1 \mathrm{hr}$ (lane 2), $4 \mathrm{hrs}$ (Iane 3 ) and 24 hrs (lane 4). Ku DNA binding activity increases 10 times after $4 \mathrm{hrs}$ of SST treatment. Signals in the autoradiogram are proportional to the Ku DNA binding. Mobility shift assay (EMSA) was performed as described in materials and methods. The experiment was repeated three times and provided similar results. The lower panels show the scanning densitometric analysis of the bands corresponding to the Ku DNA binding activity in nuclear extracts. The values on axis $y$ are means \pm s.d. of three independent experiments. Panel B: supershift of the Ku 70/86 heterodimer performed by the addition of the antiKu 70 (lane 2) or anti-Ku 86 (lane 3) policlonal antibody. Lane $1 \mathrm{Ku} 70 / 86$ DNA binding in nuclear extracts from cells SST stimulated cells, lane $p$ refers to the free probe.
(Figure 3, panel C). Infact the anti Ku86 antibody recognized in the extracts from treated cells, only the upper band in common with the anti-Ku70 staining and a second band, not cross reactive with Ku70, corresponding to the monomer form.

\section{The modulation of Ku70/86 nuclear DNA binding activity correlates to Ku86 nuclear level}

To determine whether the modulation of Ku heterodimer formation was related to a variation of p70 and p86 protein levels or to a different activation of the two subunits, nuclear and cytoplasmic level of the two subunits were examined by western blot analysis. Goat polyclonal anti-Ku70 and antiKu86 antibodies no cross-reactive were used to determine the protein levels. Figure 4 shows the p86 and p70 nuclear and cytoplasmic expression in untreated and SST treated cells at different times of stimulation. The protein level of $p 70$ seems to be not affected by somatostatin treatment either in the nucleus either in the cytoplasm. Conversely Western blot analysis showed an evident increase (2.1 time) of nuclear p86 protein levels only after 4 hrs of treatment. This result was also confirmed by immunoistochemistry on Caco cells seeded at the same density and treated with somatostatin for 4 and 24 hours (Figure 4). After 4 hours SST induced an evident increase of p86 in the nucleus in agreement with data obtained by western blot analysis (Figure 4). At 24 hours of treatment the expression and the localization of p86 was similar to that

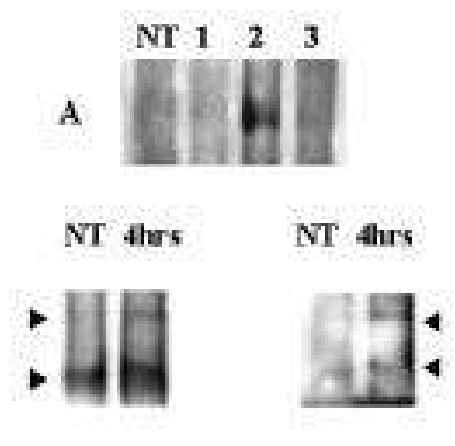

B

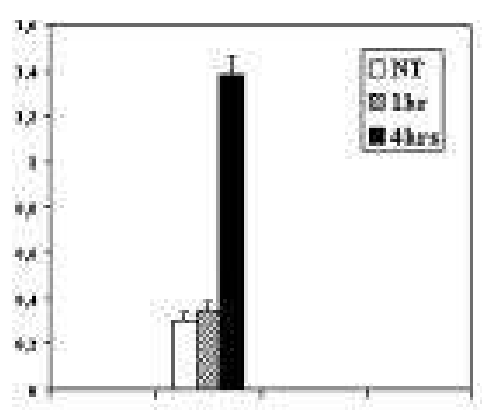

D
Figure 3. Panel A: Immunoprecipitation of nuclear extracts from Caco cell line untreated (NT) and SST treated for 1 hour (lane 1), 4 hours (lane 2) and 24 hours (lane 3). Nuclear proteins $(100 \mu \mathrm{g})$ were immunoprecipitated with $2 \mu \mathrm{g}$ of anti-Ku70 antibody as described in matherials and methods. After blotting the coimmunoprecipitated form of Ku 86 was detected by anti-Ku86 antibody. Panel B and C: native nuclear proteins from untreated (NT) and 4hours SST treated cells were resolved in a 7\% discontinuos native PAGE and analyzed by Western blot. The monomeric form of Ku70 (lower arrow in panel B) and the heterodimeric form (upper arrow, panel B) were identified using the policlonal antibody anti-Ku70. In panel C monomeric and hetherodimeric form of Ku86 nuclear protein. The upper band recognized by anti-Ku70 and anti-Ku86 antibodies correspond to the heterodimer complex. Panel D: effect of somatostatin treatment on the level of Ku 86 specific mRNA. The scanning densitometric analysis is reported by histograms, values are the means ( \pm s.d.) of three indipendent experiments. 

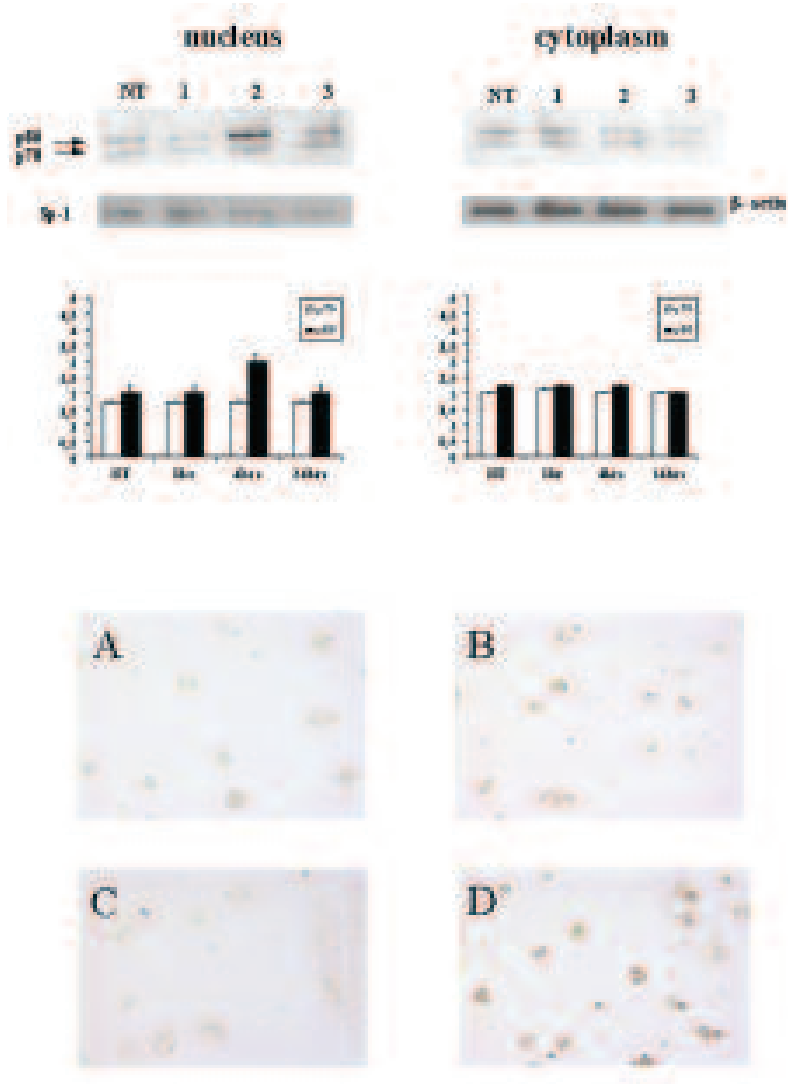

Figure 4. Western blot analysis of p70, p86 in the nuclear and in cytoplasmic extracts in untreated cells (NT) and cell treated for 1 hour (lane 1), 4 hours (lane 2), 24 hours (lane 3). Histograms represent the scanning densitometric analysis of western blot performed in nuclear extracts (left side) and cytoplasmic extracts (right side). The protein were blotted onto nitrocellulose filter and the protein expression was determined as indicated in Materials and methods. Each value was normalized for $\mathrm{Sp}-1$ or $\beta$-actin protein levels and rapresent the mean ( $\pm S D)$ of three independent experimets. Panels A, B, C, D immunocytochemistry with p86 policlonal antibody on Caco cells: untreated cultures (panel A), SST treated cells for 4 hour (panel B), for 24 hours (panel C) and for 28 hrs (24 hrs plus 4 hrs, Panel D) see Materials and Methods and Results for details.

obtained in untreated cells (panel C). On the other hand a second 4 hrs treatment, following the 24 hrs incubation, induced a strong increase of $p 86$ nuclear level comparable to that obtained with a single 4 hrs treatment (Figure 4, panel D and B).

\section{Ku86 mRNA level is affected by somatostatin treatment}

Total mRNA was extracted from Caco cells untreated and treated with somatostatin for $\mathrm{lhr}$ and $4 \mathrm{hrs}$ as described in materials and methods. After $1 \mathrm{hr}$ of stimulation no evident changes in the Ku86 mRNA level were present. Conversely after 4 hrs the SST treatment induce a strong increase of specific
Ku86 mRNA. As shown in Figure 3 panel D after 4 hrs of treatment the level of Ku86 specific mRNA was 4.67 times increased if compared to the level obtained in untreated and 1 hrs treated cells. The Ku86 mRNA level was referred to an house keeping gene as an internal control as described in materials and methods. This result indicate that the level of Ku86 specific mRNA is was affected by SST binding.

\section{Discussion}

The present study shows that somatostatin inhibition of Caco cell proliferation is related to the modulation of Ku70/86 activity, heterodimer formation and to an increase of level and expression the Ku86 mRNA. In particular somatostatin treatment modulates the nuclear Ku70/86 heterodimer activation increasing the level of Ku86 in the nucleus without affecting significatively Ku70 protein level.

Compelling evidences demonstrate that the $\mathrm{Ku}$ DNA binding activation induced by irradiation or chemical agents lead to cell accumulation in G0-G1 phase suggesting the role of $\mathrm{Ku}$ protein as a possible regulator of DNA repair and cell cycle progression (Jackson S.P., et al., 1995). According to these data, after 4 and 6 hrs of treatment, our flow cytometry studies on SST treated cells show an accumulation in G0-GI phase. The concomitant strong increase of DNA binding activity of Ku70/86 in the nucleus points out $\mathrm{Ku}$ as a mediator of somatostatin effect on cell cycle progression.

A further mechanism through which somatostatin and Ku86 cooperate in regulating the cell cycle progression seems to consist in the modulation of the PP2A mediated dephosphorilation of histone $\mathrm{HI}$, essential for chromosome condensation and DNA synthesis (Le Romancer, M. et al., 1994).

Although several studies have shown that SST influences the trafficking of the Ku subunits through the cellular compartments, the mechanisms of $\mathrm{Ku}$ activation are still unclear.

At 4 hours of treatment we observed a 10 fold increase of Ku DNA binding activity in the nucleus as compared to the untreated controls without any changes in the p70 levels. These results suggest a possible role for somatostatin in the modulation of the $\mathrm{Ku}$ heterodimer formation, possibly inducing a translocation from the membrane to the nucleus of the p86 subunit which, as previously demonstrated, 
behaves like a high affinity somatostatin receptor (Le Romancer M. et al., 1994).

There are few evidences that Ku70/86 subunits are able to translocate from the cytoplasm to the nucleus and vice versa in response to different external growth factors including EGF (Bandyopadhyay D., et al., 1998, Prabhakar B.S. et al. 1990, Teoh G. et al., 1998). In agreement with these studies, the increase of p86 levels and of Ku DNA binding activity in the nucleus, as early as at 4 hrs from the beginning of treatment, could indicate the existence of a possible cross talking among growth regulating factors of the extra cellular milieu, nuclear factors involved in cell cycle progression and DNA synthesis.

In the present study we also demonstrate that somatostatin influences the Ku86 mRNA level after 4 hours of SST stimulation. These results indicates that somatostatin could induce an increase in the transcription or in the half life of Ku86 mRNA level determining an higher expression of p86 that under SST stimulation, migrates to the nucleus and influences Ku70/86 heterodimeric activity. These data are in agreement the hypothesis that Ku86 could be a signal transducer and activator factor behaving as the intermediate of the a SST transduction pathway by the internalisation and the migration from the cell membrane to the nucleus (Boyle WJ. Et al., 1992, Le Romancer, M. et al., 1994) like other signal transducers as the members of STAT family (Kisseleva T. et al. 2002). Moreover these results point out that SST up-regulates the expression of Ku86, one of its specific receptor, inducing an increase of Ku86 mRNA level.

Our findings are consistent with the hypothesis that somatostatin regulates cell cycle progression and DNA repair influencing the Ku70/86 activity in the nucleus trough the modulation of nuclear p86, the limiting factor in the heterodimer formation, by increasing the level of Ku86 mRNA.

\section{References}

Bandyopadhyay D, Mandal M, Adam L, Mendelsohn J, Kumar R. Physical interaction between epidermal growth factor receptor and DNA-dependent protein kinase in mammalian cells. J Biol Chem 1998;273:1568-73.

Blunt T, Finnie NJ, Taccioli GE, Smith GC, Demengeot J, Gottlieb TM. Defective DNA-dependent protein kinase activity is linked to $V(D) J$ recombination and DNA repair defects associated with the murine scid mutation. Cell 1995;80:813-23.

Boyle WJ. Growth factors and tyrosine kinase receptors during devel- opment and cancer Curr Opin Oncol 1992;4:156-62.

De Vita VT Jr, Hellman S, Rosemberg SA, eds. Principles and practice of oncology. Cancer 1997; $5^{\text {th }}$ edition. Philadelphia (PA), USA.

Difilippantonio MJ, Zhu J, Chen HT, Meffre E, Nussenzweig MC, Max $E E$, et al. DNA repair protein Ku80 suppresses chromosomal aberrations and malignant transformation. Nature 2000;404:510-4.

Dignam JD, Lebovitz RM, Roeder RG. Accurate transcription initiation by RNA polymerase II in a soluble extract from isolated mammalian nuclei. Nucl Acids Res 1983;11:1475-89.

Fewell JW, Kuff EL. Intracellular redistribution of Ku immunoreactivity in response to cell-cell contact and growth modulating components in the medium. J Cell Sci 1996; 109:1937-46.

Gottlieb TM, Jackson SP. The DNA dependent protein kinase: requirement for DNA ends and association with Ku antigen. Cell 1993; 72:131-42.

Jackson SP, Jeggo PA. DNA double-strand break repair and V(D)J recombination: involvement of DNA-PK. Trends Biochem Sci 1995; 20:412-5.

Goudelock D, Kecheng J, Pereira E, Russel B Sanchez Y. Regulatory interactions between the checkpoint kinase Chk1 and the proteins of the DNA-PK complex. J Biol Chem 2003;278.

Kasten U, Plottner N, Johansen J, Overgaard J, Dikomey E. Ku70/86 gene expression and DNA dependent protein kinase (DNA-PK) activity do not correlate with double-strand break (dbs) repair capacity and cellular radiosensitivity in normal human fibrobroblast. $\mathrm{Br} \mathrm{J}$ Cancer 1999;79:1037-41.

Killeen A A. Molecular Pathology Protocols 2001; Humana press.

Kinzler KW, Vogelstein B. Gatekeepers and caretakers. Nature 1997; 386:761-3.

Kisseleva T, Bhattacharya S, Braunstein J, Schindler CW. Signaling through the JAK/STAT pathway, recent advances and future challenges. Gene 2002;20:285:1-24.

Le Romancer M, Reyal-Desmars F, Cherifi Y, Pigeon C, Bottari S, Meyer 0 , Lewin $\mathrm{M}$. The $86-\mathrm{kD}$ sibunit of autoantigen $\mathrm{Ku}$ is a somatostatin receptor regulating protein phosphatase-2A activity. J Biol Chem 1994; 269:17464-8

Li GC, Ouyang H, Li X, Nagasawa H, Little JB, Chen DJ. Ku70: a candidate tumor suppressor gene for murine $T$ cell lymphoma. Mol Cell 1998;2:1-8.

Nussenzweig A, Chen C, Da Costa-Soares V, Sanchez M, Sokol K, Nussenzweig MC. Requirement for Ku80 in growth and immunoglobulin $V(D) J$ recombination. Nature 1996;382:551-5.

Nussenzweig A, Sokol K, Burgman P, Li L, Li GC. Hipersensitivity of Ku80-deficient cell lines and mice to DNA damage : The effect of ionizing radiation on growth, survival, and development. Proc Natl Acad Sci USA 1997; 94:13588-93.

Ornstein L, Davis BJ. Ann NY Acad Sci 1964;121:321.

Prabhakar BS, Allaway GP, Srinivasappa J, Notkins AL. Cell surface expression of the 70-kDa component of $\mathrm{Ku}$, a DNA-binding nuclear autoantigen. J Clin Invest 1990;86:1301-5.

Pucci S, Mazzarelli P, Rabitti C, Giai M, Gallucci M, Flammia G, et al. Tumor specific modulation of Ku70/80 DNA binding activity in breast and bladder human tumor biopsies. Oncogene 2001;20:73947.

Sato T, Akiyama F, Sakamoto G, Kasumi F, Nakamura Y. Accumulation of genetic alterations and progression of primary breast cancer. Cancer Res 1991;51:5794-9.

Teoh G, Urashima M, Greenfield EA, Nguyen KA, Lee JF, Chauhan D. The $86-\mathrm{kD}$ subunit of $\mathrm{Ku}$ autoantigen mediates homotypic and heterotypic adhesion of multiple myeloma cells. J Clin Invest 1998; 101:1379-88.

Wilson CR, Davidsonn SE, Margison GP, Jackson SP, Hendry JH. Expression of Ku70 correlates with survival in carcinoma of the cervix. Br J Cancer 2000;83:1702-6.

Yamada Y, Post SR, Wang K, Tager HS, Bell GL, Seino S. Cloning and functional characterization of a family of human and mouse somatostatin receptors expressed in brain, gastrointestinal tract, and kidney. Proc Natl Acad Sci USA 1992;89:251-5.

Yang CR, Yeh S, Leskov K, Odegaard E, Hsu HL, Chang C. Isolation of Ku70-binding proteins (KUBs). Nucleic Acids Res 1999;27:216574. 